\title{
Ideal free flows of optimal foragers: Vertical migrations in the ocean
}

\author{
Uffe Høgsbro Thygesen, Maksim Mazuryn \\ Technical University of Denmark \\ Department of Applied Mathematics and Computer Science \\ Building 303 B , DK-2800 Kgs. Lyngby, Denmark \\ $\{$ uhth, makm\}@dtu.dk
}

October 6, 2021

\begin{abstract}
We consider the collective motion of animals in time-varying environments, using as a case diel vertical migration in the ocean. The animals are distributed in space such that each animal moves optimally, seeking regions which offer high growth rates and low mortalities, subject to costs on excessive movements as well as being in regions with high densities of conspecifics. The model applies to repeated scenarios such as diel or seasonal patterns, where the animals are aware of both current and future environmental conditions. We show that this problem can be viewed as a differential game of mean field type, and that the evolutionary stable solution, i.e. the Nash equilibrium, is characterized by partial differential equations, which govern the distributions and migration velocities of animals. These equations have similarities to equations that appear in the fluid dynamics, specifically the Euler equations for compressible inviscid fluids. If the environment is constant, the ideal free distribution emerges as an equilibrium. We illustrate the theory with a numerical example of vertical animal movements in the ocean, where animals are attracted to nutrient-rich surface waters while repulsed from light during daytime due to the presence of visual predators, aiming to reduce both proximity to conspecifics and swimming efforts. For this case, we show that optimal movements are diel vertical migrations in qualitative agreement with observations.
\end{abstract}

Keywords: Vertical migration, optimal behavior, evolutionary games, habitat selection.

\footnotetext{
${ }^{*}$ Corresponding author. ORCID: 0000-0002-4311-6324
} 


\section{Introduction}

Animals move in space in order to forage, avoid predators, or seek mates, and the resulting movements impact the dynamics of ecosystems (Nathan et al., 2008). The spatial distributions of populations, which emerge from the movements of individuals, govern the rate with which predators meet prey, and therefore how biomass is transported from lower trophic levels to higher trophic levels, as well as the growth rate and mortality of the various populations. In turn, the movements of individual animals are motivated by the spatial structure of their habitat.

A noteworthy example of animal movement is vertical migrations, which are ubiquitous in the ocean (Brierley, 2014) and are believed to constitute the largest movement of biomass on the planet. Even phytoplankton display vertical migration (Wirtz and Smith, 2020), while diel migrations of zooplankton and forage fish give rise to the dynamics of the deep scattering layer (Cisewski et al., 2010). Top predators generally track the movements of their prey, but take into account the limits imposed by physiological constraints such as light, oxygen and temperature (Thygesen et al., 2016). These migrations have implications not just for the animals that perform them, their prey and predators, but also for ecosystem functioning such as the vertical transport of carbon, a mechanism known as the "biological carbon pump" (Longhurst and Harrison, 1989). Vertical distributions also affect measurements taken from the system and their interpretation, whether trawl surveys or acoustic surveys (Gauthier and Rose, 2005).

It is generally agreed that prey descend at day into the dark deep to avoid visual predation (Bollens and Frost, 1989), and that predators follow their prey (Josse et al., 1998). While these motions therefore seem to have "bottomup" dynamics, in that the prey initiate the movement and the predators follow, they must clearly be understood in a game-theoretic setting, since they involve the simultaneous decisions of several agents which each pursue their own interests. One phenomenon which adds credibility to the gametheoretic understanding is that the migrations can drive reverse migrations by even lower trophic levels (Ohman et al., 1983): Here, as predatory copepods descend at dawn to avoid visual predation from forage fish, their smaller planktonic prey ascend to spend the day near the surface, where the absence of predatory copepods implies relative safety.

To understand and explain these vertical migrations using the notions of game theory, Iwasa (1982) was seminal in modeling the phenomenon as a matrix game. This framework was pursued further by several authors including Sainmont et al. (2013) and Pinti and Visser (2019), with increasing fidelity as well as complexity, both in terms of modeling and compuations. Thyge- 
sen and Patterson (2018) modeled the phenomenon in continuous space and time, using differential equations. This leads to higher fidelity and in particular resolves the narrow windows of opportunity and risk that arise at dawn and dusk, and also allows a more direct comparison to data. However, the modeling framework is more technically challenging, and to make progress, Thygesen and Patterson (2018) ignored the costs and constraints of locomotion. While this may be reasonable for larger animals, such as tunas, smaller animals such as zooplankton are constrained by their maximum swimming speed and the cost of locomotion can be expected to be a significant in their energy budgets.

Therefore, this study aims to improve on the modeling framework developed by Thygesen and Patterson (2018) by including the cost of locomotion. Thus, we formulate a game, where each player is an animal which chooses a trajectory in space such as to maximize its fitness in a Darwinian sense. We assume that there are effectively infinitely many players, so that model is a differential mean field game in the sense of Lasry and Lions (2007). To achieve a simple and tractable model structure, we focus on the singlespecies case, where individuals of the same species play against each other. A model with some structural similarity has recently been posed for the collective motion of human pedestrians (Achdou and Lasry, 2019).

We apply calculus of variations (Liberzon, 2011) to reach a characterization of the Nash equilibrium in terms of partial differential equations. This equations have similarities to - but also notable differences from - the Euler equations of fluid mechanics. Similar observations have been made in the field of differential mean field games (Lasry and Lions, 2007); the underlying explanation is that fluids can be seen as a continuum of molecules, which each satisfy the principle of least action (Goldstein et al., 2002).

Our project is thus to pose partial differential equations that govern the abundance of animals. Such models are common in mathematical ecology (Okubo and Levin, 2001). However, the majority of such models are parabolic and describe how animals move in a preferred direction (display taxis) while at the same time spread according to a diffusive process, to account for the empirical fact that the details of animal motion is unpredictable. These models lead to initial value problems. In contrast, the equations in this study are hyperbolic, and the preferred directions and speeds of the animals are established as part of the solution. They do not address the randomness, since each animal is assumed to be a perfect decision maker, and the reason animals distribute themselves over space is not diffusive processes but rather density dependence of fitness. Further, the models in this paper should not be posed as initial value problems, since we assume that the animals move in predictable environments and are able to adjust in advance to anticipated events, such as sunset. In general, they 
must be addressed as problems with mixed initial and terminal conditions, as is in the typical case in dynamic optimization (Liberzon, 2011). Here, our focus is on periodic solutions that describe daily patterns, ignoring the slower timescales of seasonality as well as population dynamics.

An important element of our contribution is numerical analysis of the resulting equations. The structural similarity between the dynamics of fluids and those of optimal foragers suggest to find inspiration in well-established methods for computational fluid dynamics. However, these methods cannot be used off the shelf, since we pursue periodic solutions which are unstable, and therefore we establish numerical methods tailored for the specific problem.

\section{Migrations as a dynamic game between individ- uals of the same species}

Consider a population of animals distributed in a spatial domain $\mathbf{X} \subset \mathbf{R}^{d}$; the dimension $d$ of the space may be one, two or three. We are interested in the density of the animals, i.e. the function $N(t, x), t \in \mathbf{R}, x \in \mathbf{X}$. Animals at position $(t, x)$ move vertically with velocity $V(t, x)$. Our current aim is to pose equations that govern $N$ and $V$. We ignore population dynamics, so that the continuity equation

$$
\dot{N}+\nabla \cdot(N V)=0
$$

holds. Here, $\dot{N}=\partial N / \partial t$ is time derivative while $\nabla \cdot$ is the divergence, $\sum_{i} \partial / \partial x_{i}$. Our interest is in bounded domains with no-flux boundary conditions:

$$
(N V)(t, x) \perp n(x) \text { for all } t \in \mathbf{R}, x \in \partial \mathbf{X}
$$

where $n(x)$ is normal to the boundary of $\mathbf{X}$ at $x$. The velocity field $V$ arises from decisions by the animals and thus derives from fitness optimization. To detail this, animals at time $t$, position $x$ and with velocity $v$ have instantaneous rate of energy harvest $g(t, x)$ and energy expenditure on locomotion $\frac{1}{2} \nu|v|^{2}$. We also assume a density-dependent mortality

$$
\mu(t, x)=\mu_{0}(t, x)+\mu_{1} N(t, x)
$$

which is large enough to affect the decisions of the animals yet small enough that the resulting population dynamics can be ignored. Our main interest is the situation where $g$ and $\mu_{0}$ are periodic in time with period $T=1$ day. Let $F$ be the fitness of an animal, defined as the expected harvest of energy until the animal dies. Since we assume that the mortality is small enough that population dynamics can be ignored, we also ignore fluctuations in fitness 
over the single day and consider $F$ a constant parameter. Define the net gain rate

$$
r(t, x, v)=g(t, x)-\mu(t, x) F-\frac{1}{2} \nu|v|^{2},
$$

which includes harvest, cost of locomotion, and loss of fitness due to mortality. If an animal chooses a trajectory $\left\{X_{t}: t \in[0, T]\right\}$ with velocity $\left\{U_{t}=d X_{t} / d t=V\left(t, X_{t}\right)\right\}$, then its change in fitness over one period $[0, T]$ is

$$
\int_{0}^{T} r\left(t, X_{t}, U_{t}\right) d t
$$

Our fundamental assumption is that all animals behave optimally in the sense of maximizing this integral. I.e., the solution $(N, V)$ must have the property that no animal can benefit by deviating from the strategy $U_{t}=$ $V\left(t, X_{t}\right)$. From an evolutionary point, this amounts to the strategy $U_{t}=$ $V\left(t, X_{t}\right)$ being evolutionary stable. From a game-theoretic perspective, it implies that the system is at a Nash equilibrium of the mean field game, where each individual plays against the continuum of the remaining population.

From this fundamental assumption, we can derive the variational principle that the change in fitness (5) over one period must be insensitive to perturbations $\delta X_{t}$ of the trajectory; i.e., the first variation vanishes. As is standard in calculus of variations (Liberzon, 2011), we reach the Euler-Lagrange equations, which are $d$ coupled ordinary differential equations governing $X_{t}$ and $U_{t}$ :

$$
\frac{\partial r}{\partial x}\left(t, X_{t}, U_{t}\right)-\frac{d}{d t}\left[\frac{\partial r}{\partial v}\left(t, X_{t}, U_{t}\right)\right] d t=0 .
$$

Inserting the specific forms for $r$, we find

$$
\nabla\left(g-F \mu_{0}\right)-\mu_{1} F \nabla N+\nu \frac{d}{d t} U_{t}=0
$$

Here, we omit the arguments $\left(t, X_{t}\right)$ for notational clarity. If an animal has velocity $U_{t}=V\left(t, X_{t}\right)$, then its acceleration is $\frac{d}{d t} U_{t}=\nabla V\left(t, X_{t}\right) V\left(t, X_{t}\right)+$ $\dot{V}\left(t, X_{t}\right)$, where $\nabla V$ is a matrix field with elements $\partial V_{i} / \partial x_{j}$. We obtain:

$$
\nu[\dot{V}+(\nabla V) V]+\nabla\left(g-F \mu_{0}\right)-\mu_{1} F \nabla N=0 .
$$

Importantly, this equation must only hold where animals are found, i.e. at points $(t, x)$ such that $N(t, x)>0$. In places $(t, x)$ where $N(t, x)=0$, the velocity is not defined, but any trajectory that spends time in a region with $N=0$ must lead to an increase in fitness over a period which is no greater. In this study we disregard this side condition, as our numerical case has $N(t, x)>0$ everywhere, but we shall return to it in the discussion. 
It is instructive to notice the structural similarity betwen this equation (6) and the one describing conservation of momentum in the Euler equations governing flow of compressible inviscid fluids (Batchelor, 1967); see also the discussion in (Lasry and Lions, 2007). In this analogy, the term $g-\mu F$ corresponds to a potential from external forces that drive the motion. Notice that the resulting "force" on the individual animal is in the direction of decreased harvest rate $g$ and increased mortality $\mu_{0}$. This may seem counter-intuitive, but is consistent with the single-particle problem (Thygesen, in prep). The term $\mu_{1} F \nabla N$ loosely corresponds to the pressure term in fluid dynamics. A precise analogy is obtained if we define the "pressure" as $p(t, x)=-N^{2}(t, x) \mu_{1} F / \nu$. Note the sign and that the "force" on the individual animal, arising from the pressure gradient, is towards increased densities, which also may seem counter-intuitive but follows the same logic as the external forces. The underlying reason for the similarity between the dynamics of fluids and those of optimal foragers is the principle of least action (Goldstein et al., 2002). This principle, which permeates physics, states that the microscopic particles that constitute a fluid follow trajectories which render the so-called action integral stationary, in the same way optimal foragers follow trajectories which render the fitness integral stationary.

The fitness $F$ is a parameter in these equations. Internal consistency would require that $F$ derives from the integral of $r$, but with the same appeal to the quasi-stationary approximation as in the previous, we ignore this constraint: Our interest is in the behavior during a single day at a given time of the year, and the fitness at any given day depends on fluctuations in conditions over the year. Therefore, we can consider $F$ a free parameter.

\subsection{Steady state and the ideal free distribution}

Our principal interest is on the dynamics, but it is useful to also consider steady states. This, of course, assumes that the harvest rate $g$ and the mortality $\mu$ do not vary with time. For simplicty, we consider one spatial dimension $(d=1)$. Then, we seek solutions where $\partial N / \partial t=0$ and $V \equiv 0$, so that

$$
\frac{\partial g}{\partial x}-\frac{\partial \mu_{0}}{\partial x} F-\mu_{1} F \frac{\partial N}{\partial x}=0
$$

By integration, we find $g=\mu_{0} F+\mu_{1} F N+c$ where $c$ is an integration constant. In a true steady state, consistency requires that the fitness $F$, which is the expected future harvest in the remaining lifetime of an individual, satisfies

$$
F=\frac{g(x)}{\mu_{0}(x)+\mu_{1}(x) N(x)}
$$


for all $x$. Thus the integration constant vanishes $(c=0)$, and we reach

$$
N(x)=\frac{g(x)-F \mu_{0}(x)}{F \mu_{1}} \text { whenever } N(x)>0 .
$$

This is the celebrated ideal free distribution (Fretwell and Lucas, 1969). We can therefore view our model as an extension of the ideal free distribution to dynamic situations and taking into account cost of locomotion. If the total abundance, i.e. the integral $\int N(x) d x$, is known, then this allows us to determine $F$ and subsequently the distribution $N(x)$. Alternatively, if we assume steady-state also for population dynamics, then the fitness $F$ can in principle be determined as the energetic cost to produce an offspring, and this allows us to determine the distribution $N(x)$ and the total abundance $\int N(x) d x$.

This establishes an equilibrium solution to the governing equations (1), (6) for the case where the parameters $g$ and $\mu$ are constant in time. It is possible to show that this equilibrium is hyperbolic; i.e., there exist stable and unstable manifolds consisting of solutions that converge to, or diverge from, the equilibrium. An important consequence of this, for the case of timevarying parameters, is that one should not attempt to solve the governing equations as initial value problems: Such solutions will quickly diverge along the unstable manifolds, which renders the solutions useless. This is a general feature of dynamic optimization problems (Liberzon, 2011).

\section{A numerical example}

In this section we discuss a particular case of diel vertical migration of an aquatic species. The model is chosen to illustrate the mathematical framework as simply as possible, rather than to mimic a specific system. We envision a species of zooplankton, e.g. copepods, that are small enough that their movements are constrained by viscous drag and hence the power required is quadratic in the speed, in agreement with (6). We consider a shallow habitat such as a lake or a continental shelf sea, so that it is plausible that the simplifying condition $N(t, x)>0$ holds. The copepods are subject to predation by planktivorous fish, which rely on visual detection, so that the instantaneous mortality depends on local light levels. The copepods themselves, in turn, rely on mechanosensing, so that their feeding rates do not depend on light levels but only on the abundance of prey, which varies with depth but not with time. 


\begin{tabular}{clrc}
\hline Symbol & Parameter & Value & Units \\
\hline$H$ & Depth & 200.0 & $\mathrm{~m}$ \\
$T$ & Period & 24.0 & $\mathrm{hour}$ \\
$A$ & Amplitude of light fluctuations & 10.0 & - \\
$\nu$ & Drag & 1.0 & $\mathrm{Jh} / \mathrm{m}^{2}$ \\
$\mu_{1}$ & Density-dependent mortality & 0.5 & $1 / \mathrm{hour}$ \\
$F$ & Fitness & 10 & $\mathrm{~kJ}$ \\
$k_{1}$ & Absorption coefficient & 0.05 & $1 / \mathrm{m}$ \\
$g_{0}$ & Maximum food availability & 100.0 & $\mathrm{~J} / \mathrm{hour}$ \\
$k_{2}$ & Harvest decay rate & 0.02 & $1 / \mathrm{m}$ \\
$m$ & Mortality scaling & 5.0 & $\mathrm{~m}^{-2} \mathrm{~s}^{-1}$ \\
$I_{0}$ & Maximum irradiance & 1.0 & $\mathrm{kilolux}$ \\
$K$ & Saturating light level & 1.0 & kilolux \\
\hline
\end{tabular}

Table 1: Parameters in the model.

\subsection{Model specification}

We now detail the model, i.e., the functional forms of energy harvest rates and mortalities. Specific parameters in the model are given in table 1 and argued for at the end of this section.

\section{Mortality}

The density-independent mortality $\mu_{0}$ stems from visual predators. We take the resulting mortality to derive directly from the local light intensity, which governs the distance at which a predator can detect its prey. Our starting point is therefore the surface irradiance, which varies with the time of day according to the periodic function

$$
I_{s}(t)=\frac{I_{0}}{1+\exp (A \cos (2 \pi t / T))}
$$

The resulting irradiance is seen in figure 1 (left panel). A more elaborate model would take into account astronomy and atmosphere optics, as done by Thygesen and Patterson (2018), but such an effort would hardly be worthwhile in the present more idealized context. Below the water surface we assume that the light abundance decays exponentially with absorption coefficient $k_{1}$. Then the light intensity $I(t, x)$ at depth $x$ is given by

$$
I(t, x)=I_{s}(t) \exp \left(-k_{1} x\right) \quad .
$$


We next introduce the local detection radius $r(t, x)$, which is defined as the distance at which a predator can detect a prey at depth $x$ and time $t$. We follow the reasoning of Aksnes and Utne (1997) and take as detection criterion that enough scattered photons from a prey arrive on the predator's retina and trigger a neural response in the predator. Aksnes and Utne (1997) take into account the spherical spread of light between prey and predator, the exponential decay of light along this line due to absorption, and saturation in the neural response, and arrive at the following transcendental equation

$$
r^{2} \exp \left(k_{1} r\right)=\frac{I(t, x)}{K+I(t, x)},
$$

where $I$ is the light level from (8) and $K$ is a saturation parameter reflecting the transformation of the light energy to neural activity. Once the detection distance $r$ has been found, we posit that the predation mortality scales quadratically with the detection distance:

$$
\mu_{0}(t, x)=m r^{2} .
$$

The justification is that predators cruise through the water with a speed that exceeds those of the zooplankton, effectively clearing cylindrical volumes (Kiørboe, 2008) in which the radius is $r$. This implicitly assumes that the density of predators is constant; a simplification that could be bypassed by explicitly modeling the distribution and movements of predators. The parameter $m$ combines the speed, abundance and attacking success of predators. The resulting mortality is depicted in figure 1, right panel.

The density-dependent mortality, in turn, is considered constant and fixed (table 1). This is a coarse simplification of how densities of conspecifics affect the vital rates of an organism, and its justification is solely mathematical simplicity. We return to this in the discussion.

\section{Energy harvest rate}

We assume that the energy harvest rate is greatest near the surface and decreases with depth. Since we do not wish to include an explicit model of the distribution of the phytoplankton that are the food source of our focal organism, we simply assume that harvest rate $g(t, x)$ decreases exponentially with depth:

$$
g(t, x)=g_{0} \exp \left(-k_{2} x\right) \quad .
$$

Note that we take the harvest rate to be constant in time. This is consistent with copepods detecting prey using mechanical cues and assumes that behavior and prey density is constant during the day, which appears to be a reasonable approximation. 

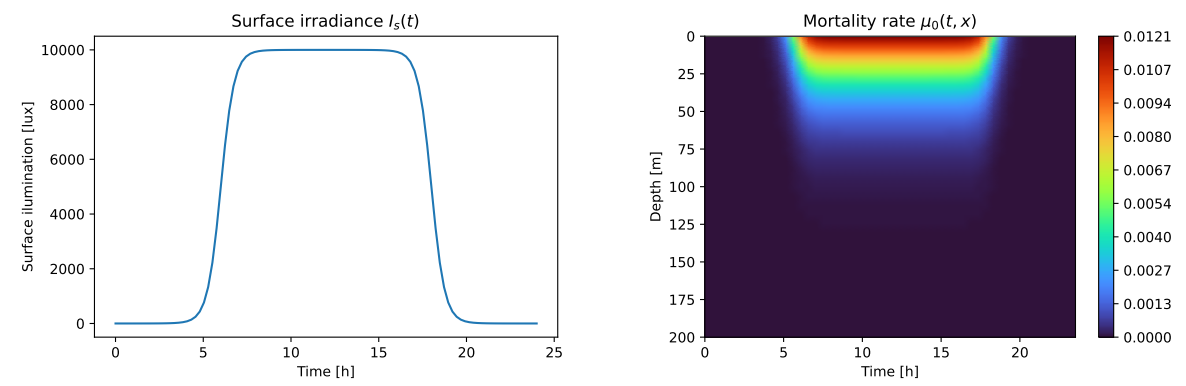

Figure 1: Model components. Left panel: Surface irradiance $I_{s}(t)$ during a day. Right panel: The density-independent mortality rate $\mu_{0}(t, x)$ as a function of depth and time of day.

\section{Model parametrization}

The numerical values for parameters, as given in table 1, are fixed so as to mimic a case of planktonic copepod of diameter $5 \mathrm{~mm}$, even if their specific values are fixed mainly for illustrative purposes. The maximum light level $I_{0}=1$ kilolux just below the surface corresponds to a typical clear day, and the light amplitude $A=10$ implies that the light levels at midnight correspond to a clear night with full moon. The day is roughly as long as the night, meaning that the scene is low latitudes and/or around equinox. The absorption coefficient $k=0.05$ per meter corresponds to clear ocean water. A saturation constant of $K=1$ kilolux in the vision of the predatory fish implies that they are generally not limited by light saturation, except partly at noon and at the surface. With these parameters, it is the spherical spread of light between prey and predator, rather than absorption, that determines the detection probability.

The fitness of the animal, which is defined as the expected future investment in reproduction, is set at $10 \mathrm{~kJ}$, corresponding to the energetic contents of 0.25 grams fat, which shall be seen relative to a body weight of roughly 0.05 grams. The drag coefficient of $1 \mathrm{Jh} / \mathrm{m}^{2}$ corresponds to a spherical body of diameter $5 \mathrm{~mm}$ and a viscosity of $1 \mathrm{mPas}$, which is roughly the viscosity of water at 5 degree Celsius.

The parameters describing mortality and growth are fixed in coarse agreement with this fitness, keeping in mind that we study a single day during the yearly cycle, so that fitness does not need to balance growth and mortality exactly. The maximum growth rate of $100 \mathrm{~J} / \mathrm{h}$ implies that the fitness of an animal corresponds to 4 days of feeding at the surface, or 11 days of feeding at 50 meters, with a decay rate of $k_{2}=0.02 / \mathrm{m}$. The parameter $m$, specifying the mortality, is fixed at $m=5 \mathrm{~m}^{-2} \mathrm{~s}^{-1}$ so that the peak mortality, 
found at surface during noon, is 0.012 per hour. Note that very few animals will be exposed to this mortality, since the animals will tend to avoid this region, and that this mortality is only found during daytime.

\subsection{Computational scheme}

We determine the periodic-in-time solution to the equations (1), (6) with noflux boundary conditions (2) numerically by discretizing time and space and solving the resulting system of algebraic equations using a Newton method. To ensure convergence of the Newton method, we apply a homotopy method. Specifically, we include a homotopy parameter $\epsilon$ which modifies the original equations (1), (6) to the following system

$$
\begin{aligned}
\frac{\partial N}{\partial t}+\frac{\partial(N V)}{\partial x} & =0 \\
\nu\left(\frac{\partial V}{\partial t}+\frac{1}{2} \frac{\partial\left(V^{2}\right)}{\partial x}\right)+\epsilon \frac{\partial g}{\partial x}-F\left(\epsilon \frac{\partial \mu_{0}}{\partial x}+\frac{\partial\left(N \mu_{1}\right)}{\partial x}\right) & =0
\end{aligned}
$$

Following the homotopy principle, the case $\epsilon=1$ recovers the original system of equations, while with $\epsilon=0$ the analytical solution is known: $N(t, x)$ is constant in space and time, and $V(t, x) \equiv 0$. Increasing the parameter $\epsilon$ from 0 to 1 , we track the solution, until the solution to the original system is obtained with $\epsilon=1$.

For a given value of $\epsilon \in(0,1]$, we find the solution using an iterative Newton method. At each iteration, we linearize the equations around the previous guess. We then solve the resulting linearized PDE system, discretizing both time and space using the spectral collocation method. As functional basis we choose the Fourier basis with equidistant quadrature nodes for time, since we pursue periodic-in-time solutions, while for space we use a Legendre basis with Gauss-Lobatto quadrature nodes, respectively (Kopriva, 2009). The computations are performed in Python.

\section{$3.3 \quad$ Results}

Figure 2 displays the densitiy $N(t, x)$ corresponding to the base parameters in table 1 (top left panel). During the daytime, the animals move to deeper waters to avoid visual predation, while at dusk they return to upper levels to benefit from better feeding conditions during the night. The panel also displays the trajectories of 3 individual animals, chosen at the 25, 50, and 75 percentile of the distribution, respectively. Animals closer to the surface undertake larger vertical migrations than those that reside deeper, which is consistent with the environmental fluctuations being larger at the surface. 


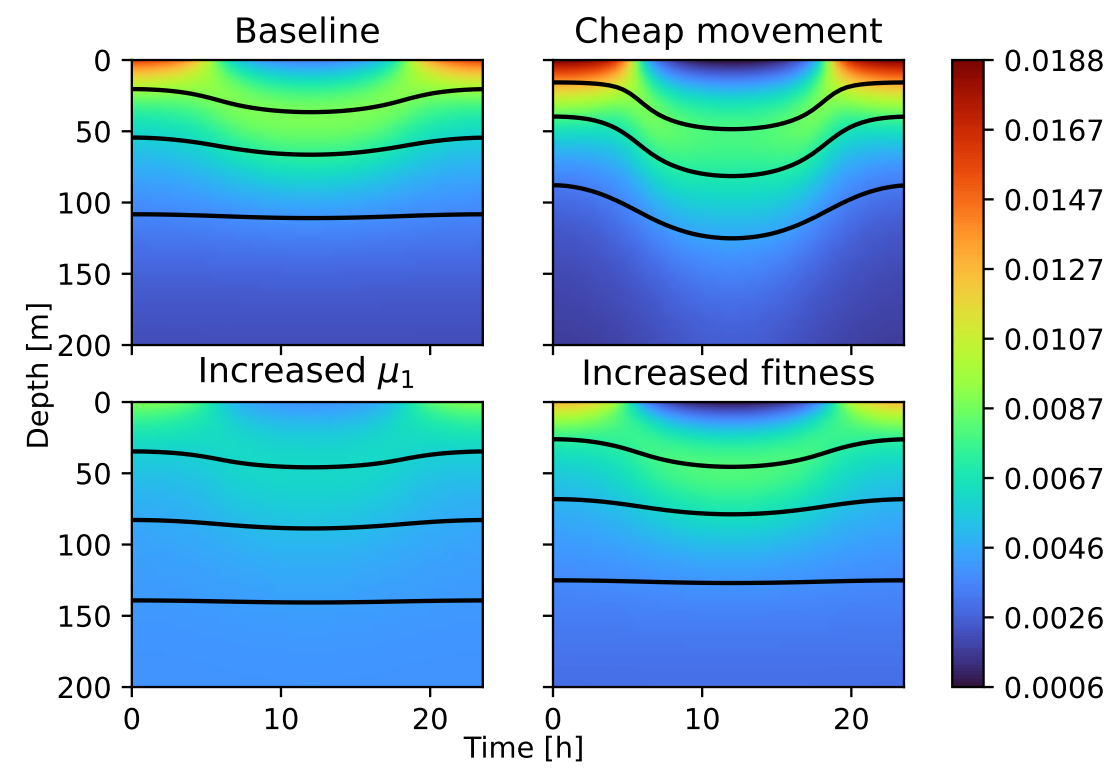

Figure 2: Different simulated scenarios. Dark lines correspond to trajectories of an animal. Top left: Parameters from 1. Top right: Decreased cost of motion $\nu(\times 0.1)$. Bottom left: Increased density dependent mortality $\mu_{1}$ $(\times 3)$. Bottom right: Increased fitness $F(\times 1.25)$.

The figure also displays results of modifying the underlying parameters. In the top right panel, the cost of motion has been decreased by multiplying the parameter $\nu$ with a factor 0.1 . As expected, the effect of this is that the animals perform migrations with broader range. In the bottom left panel, the parameter $\mu_{1}$ describing density-dependent mortality has been increased with a factor 3 , resulting in a less concentrated distribution of animals. In the bottom right panel, the fitness $F$ has been increased with a factor 1.25. The effect of this is that the trade-off between energy harvest and mortality is altered, so that the animals pursue more cautious strategies, i.e., follow deeper trajectories.

Figure 3 shows the environment that a single animal experiences during the course of a day. The quantile in these plots refer to the vertical distribution of animals, so that an animal with quantile 0 is always the closest animal to the surface while an animal with quantile 0.5 always has $50 \%$ of the animal population above it. Note that the model assumes that animals maintain their quantiles during the day, i.e. they do not pass eachother, as this would be suboptimal. In the left panel, we see the light experienced by the animals. We see that animals, despite migration downward at dawn, experience much higher light levels during the day, although the light levels 

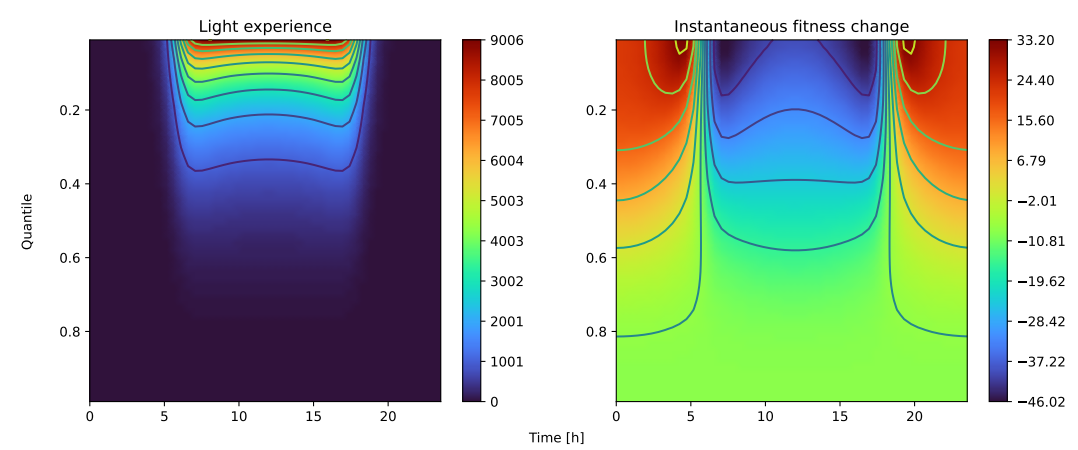

Figure 3: The environment that an animal experience during a day. In both panels, the abscissa is time during the day (in hours) while the ordinate gives the quantile of the animal in question; 0 being the animal nearest the surface and 1 being the animal nearest the bottom. Left panel: The light level experienced by the animal (in lux). Right panel: The instantaneous rate of change in the fitness of the animal. Both panels are for the reference parameters in table 1.

gradually decreases around noon, as the animals continue their migration. In the right panel, we see the instantaneous rate of change of fitness, i.e. the integrand $r$ in (5) given by (4). We see that animals at depth experience a gradual loss of fitness with a small and roughly constant rate. In contrast, the animals closer to the surface experience great daily fluctuations, where the nighttime provide positive change due to feeding opportunities under relative safety, while the daytime poses threats of mortality that outweigh feeding opportunities.

Figure 4 shows the contributions to the change in fitness, integrated over the single day, for different animals given in terms of their quantiles. The left panel shows the net growth (uptake minus cost of motion) as well as loss of fitness due to mortality, while the right panel focuses on cost of transport. Note the different scales on the two abscissa; the cost of motion is roughly two orders of magnitude lower than the other fitness components. In the left panel, we see that all animals have the same loss of fitness of approximately $250 \mathrm{~J}$ during the day, which follows from the construction of a Nash equilibrium between the animals. However, the different animals reach this total in very different ways: The deepest animals have hardly any growth and low mortality, correspoding to an expected life time of 40 days, while the animals closest to the surface only have an expected lifetime of about 4 days, but compensate with significant growth. In the right panel, we see that the cost of motion is generally low in comparison to the other components, but also that it is the animals in the middle, that spend energy 

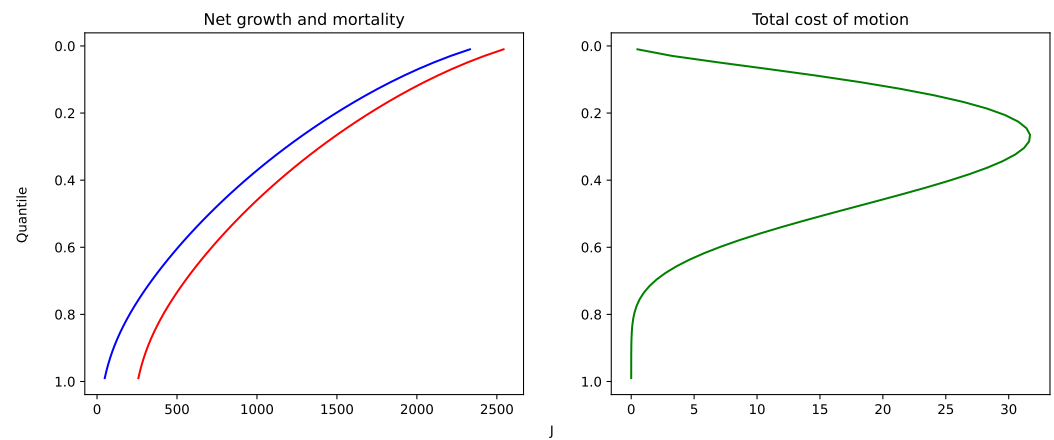

Figure 4: The components in the daily change in fitness. In both panels, the abscissa gives the change in fitness (in joules) while ordinate gives the quantile of the animal in question, as in figure 3. Left panel: Net growth (uptake minus cost of motion) (blue), and loss of fitness due to mortality (red). Right panel: Total cost of motion (green).

on migrating.

\section{Discussion}

In this paper we have considered a population distributed in space, where each individual moves such as to maximize its fitness, and shown that the governing equations for the bulk movement of the population coincides with those that describe an inviscid compressible fluid. In this analogy, the growth and mortality serves as an exogenous potential that varies in time and space and which drives the motion, while the density dependence of fitness corresponds to the thermodynamic concept of pressure. This analogy gives conceptual insight which may appeal in particular to ocean ecologists, as fluid flows already play a fundamental role in shaping oceanic habitats as well as the vital rates of animals that move and forage in these habitats. The analogy also gives access to a rich toolbox for analysis, including numerical. For example, the field of fluid mechanics has established several dimensionless quantities which are known to govern the characteristics of the resulting flow, and an interesting avenue of research would be to establish and interpret similar dimensionless quantities for the movement of animals.

Nevertheless, there are important differences between the motion of animals and those of fluid particles, even within the model. Firstly, while the potential in a physical model corresponds to net growth in a behavioral optimization model, we expect a compressible fluid to concentrate where the potential is lowest, while we expect animals to aggregate where the net 
growth rate is highest. Similarly, it may seem disturbing that the "pressure" of animals is negative and decreases with the density. These observations are related to the solutions of interest: While focus of fluid mechanics is, generally, on (Lyapunov) stable solutions or attractors, the solutions of interest for the motion of animals are intrinsically unstable when viewed as initial value problems. Indeed they should not be seen as initial value problems; the solutions are stabilized by the animals looking ahead in time when taking decisions, so information travels backwards while densities evolve forwards. This is the situation for optimal control problems (Liberzon, 2011) as well as for mean-field differential games (Lasry and Lions, 2007; Achdou and Capuzzo-Dolcetta, 2010). For numerical analysis, one can therefore not rely on time stepping (neither forwards or backwards), which is the reason we in this paper have focused on time-periodic solutions, as was also done by Achdou and Capuzzo-Dolcetta (2010). Alternatively, one may consider mixed initial/terminal value problems, as was done by Achdou and Lasry (2019) for pedestrians.

We have pointed out that when parameters are constant in time, the ideal free distribution emerges as a special solution, and thus our model is a dynamic generalization of the ideal free distribution. There are several alternative approaches to such dynamic ideal free distributions, as discussed by Cosner (2005) who establishes one such generalization. A key distinguishing element is what is assumed regarding the information available to the animals. Here, we make the idealization that animals are perfectly aware of both current and future conditions, which is in stark contrast to the assumption behind e.g. chemotaxis, where animals have to infer on local conditions from sensory inputs. Thus, our model can explain motion in response to periodic fluctuations in conditions, which have remained stable over evolutionary timescales. Diel and seasonal migrations are, of course, the most immediate examples of such motion.

In the periodic solutions we establish, all animals obtain by definition the same increase in fitness over the day, even if this increase may be distributed over the period differently for different animals. However, the animals closer to the surface obtain this increase by aggressively pursuing high growth rates, thus also enduring high mortalities, and expending high movement costs. In contrast, the deeper animals move less and have slower growth and lower mortality. Thus, the population spans a niche in which different zones call for different specialized adaptations, and it is plausible that there are limits to how wide this niche can be while still remain in the scope of the species. These considerations are beyond the present modeling study, but would be interesting to pursue.

While the solutions we have constructed represent optimal trajectories that an animal should follow, we have not adressed if and how actual animals are 
able to adopt strategies that result in these trajectories, and also if these animals agree with us as to what should be optimized. The solutions we have established resemble qualitatively the diel vertical migrations of zooplankton that have been widely documented, e.g. by Klevjer et al. (2016) and the references therein, but we have refrained from a more quantitative comparison since we have not parameterized the model for a specific time, place, and species. It is plausible that similar motions can be implemented with simple behavioral rules; for example, aiming to uphold a constant light intensity while avoiding high densities of conspecifics as well as fast movements. The motivation behind the current study is that if there is a selective advantage in pursuing a specific migration pattern, then it is plausible that evolution has somehow established a mechanism which yields resembling patterns. An interesting avenue of future research would be to investigate to which degree the optimal patterns vary with environment, thus extending the sensitivity study lying under figure 2, and to which degrees such differences can be obtained by simple behavioral rules. Such a study would arguably be more worthwhile in a more specific setting, parameterized for a particular species and system, as opposed to the idealized and generic settings of the current study.

At a technical level, we established the governing equations using calculus of variations. The most common approach in studies of mean field games is to pose a Hamilton-Jacobi-Bellman equation, which in our situation would govern the optimization problem that the individual animal faces, and in which the density of conspecifics enter as data. Here, since we do not include diffusive motion in the model, the calculus of variations seems more direct and also yields the similarity with the Euler equations of fluid dynamics. If diffusivity was included, the approach based on the Hamilton-Jacobi-Bellman equation appears stronger. Diffusivity is not required in our model, as the density dependence limits how much animals can aggregate, so in the interest of sparsity we have omitted diffusion from the model. However, without diffusivity, the model can yield regions that are completely void of animals due to disadvantageous conditions at a given point in time, in the same way the ideal free distribution in general predicts void regions. Such regions are numerically challenging, which is why we chose parameters for our numerical case study to avoid such regions. It would be interesting to investigate if such void regions can be resolved numerically using techniques from free surface flows, but pragmatically they can also be avoided by including a small diffusivity as a regularization parameter. We intend to pursue more efficient numerical methods in future studies, so that wider regions in parameter space can be explored, and in particular so that regions void or almost-void of animals can be investigated.

Our numerical analysis has employed spectral methods, while the common 
choice in numerical analysis of mean field games is finite difference methods (Achdou and Capuzzo-Dolcetta, 2010; Achdou and Lasry, 2019). The time periodicity of the domain, and the expected smoothness of the solution, motivated this choice. While spectral methods in this case give relatively high fidelity with relatively few basis functions, it is more difficult to establish properties of the discretized operators which guarantee convergence of the iterative solvers. Although the homotopy principle performed well, we believe that this issue underlies the numerical problems we encountered when pushing the limits of parameters space, in particular as regions in space become almost-void of animals. We aim to investigate this further in future studies.

Our model is built using simplified functional forms, which could be modified in future studies. First, we have assumed that the cost of movement is quadratic in the speed. This describes the energy spent on propulsion assuming that the flow around the animal is creeping, which is justifiable for small animals. Other forms could include inviscid flows, which would lead to a cubic relationship between speed and cost, or lost opportunity, as described e.g. by (Thygesen et al., 2016). A second simplification is that the cost depends linearly on the density of conspecifics. A mechanistically based density dependence is not trivial to include in the model, as it could involve both interference in the foraging of conspecifics as well as attraction of predators to patches of conspecifics. Both of these extensions point in the direction of two-species models, or more generally multi-species models, which we aim to address in future studies. In the present study, the role of density dependent is to avoid singular distributions, i.e. all animals following the same trajectory, whereas the specifics of the density dependent term is less well founded. Another simplification is the form of the nutrient uptake, i.e. the term $g$ in (4). It simple to extend this uptake to reflect a specific habitat with higher fidelity, but a more substantial extension, which would require quite different techniques for analysis, would be to include the dynamics and constraints of stomach fullness.

Our model is based on a time scale separation, where we argue that population dynamics can be ignored when studying a single day, even if mortality is high enough that animals take it into account when choosing diel strategies. It would be easy to include mortality in the conservation equation, and correspondingly making the fitness a function of time, but we would then pursue quasi-periodic solutions rather than strictly periodic ones, and the benefit of including mortality explicitly would be questionable. Reproduction would be more difficult to include, in particular the fidelity of such a model would probably also have to include seasonal fluctuations in conditions and the stage structure of the populations in question. Extending the model in these directions would perhaps be simple from a conceptual 
perspective, but tedious in terms of modelling and computations, and we believe that such an effort would not result in increased clarity compared to our approach of investigating a fast periodic pattern during which life history can be neglected.

In conclusion, we have established a framework consisting of partial differential equations governing the optimal motion of individual members of a population in response to periodically fluctuating environmental conditions. The equations are similar to those governing fluid flows, so that their solutions describe ideal free flows of optimal foragers. We have investigated a particular case, in an idealized setting, of zooplankton performing diel vertical migrations in the water column in response to sunrise and sunset, and shown that the model predicts patterns which are similar to those empirically observed. We believe that our approach of viewing migrating animals as elements in ideal free flows can illuminate animal migration, and offers rich opportunities for further development, both in terms of theoretical analysis and specific case studies.

\section{Declarations}

\section{Funding}

MM is partially funded by The Centre for Ocean Life, a VKR Centre of excellence supported by the Villum Foundation.

\section{Conflicts of interest}

The authors have no relevant financial or non-financial interests to disclose.

\section{Ethics approval}

Not applicable.

\section{Consent to participate}

Not applicable. 


\section{Consent for publication}

Not applicable.

\section{Availability of data and material}

Not applicable.

\section{Code availability}

The code used to perform the calculations and generate the figures will be placed in a publicly accessible GitHub repository after acceptance of the manuscript.

\section{Author's contributions}

UHT conceived the study and the analysis. MM detailed the analysis and the numerical implementation. UHT drafted the initial manuscript and both authors completed the manuscript.

\section{References}

Achdou Y, Capuzzo-Dolcetta I (2010) Mean field games: numerical methods. SIAM Journal on Numerical Analysis 48:1136-1162.

Achdou Y, Lasry JM (2019) Mean field games for modeling crowd motion In Contributions to Partial Differential Equations and Applications, pp. 17-42. Springer.

Aksnes DL, Utne ACW (1997) A revised model of visual range in fish. Sarsia 82:137-147.

Batchelor G (1967) An Introduction to Fluid Dynamics Cambridge Mathematical Library.

Bollens SM, Frost B (1989) Predator-induced diet vertical migration in a planktonic copepod. Journal of Plankton Research 11:1047-1065.

Brierley AS (2014) Diel vertical migration. Current biology 24:R1074-R1076. 
Cisewski B, Strass VH, Rhein M, Krägefsky S (2010) Seasonal variation of diel vertical migration of zooplankton from ADCP backscatter time series data in the Lazarev Sea, Antarctica. Deep Sea Research Part I: Oceanographic Research Papers 57:78-94.

Cosner C (2005) A dynamic model for the ideal-free distribution as a partial differential equation. Theoretical Population Biology 67:101-108.

Fretwell SD, Lucas HL (1969) On territorial behavior and other factors influencing habitat distribution in birds. Acta Biotheoretica 19:16-36.

Gauthier S, Rose GA (2005) Diel vertical migration and shoaling heterogeneity in Atlantic redfish: effects on acoustic and bottom-trawl surveys. ICES Journal of Marine Science 62:75-85.

Goldstein H, Poole C, Safko J (2002) Classical mechanics.

Iwasa Y (1982) Vertical migration of zooplankton: a game between predator and prey. The American Naturalist 120:171-180.

Josse E, Bach P, Dagorn L (1998) Simultaneous observations of tuna movements and their prey by sonic tracking and acoustic surveys. Hydrobiologia 371:61-69.

Kiørboe T (2008) A Mechanistic Approach to Plankton Ecology Princeton University Press.

Klevjer TA, Irigoien X, Røstad A, Fraile-Nuez E, Benítez-Barrios VM, Kaartvedt S (2016) Large scale patterns in vertical distribution and behaviour of mesopelagic scattering layers. Scientific reports 6:19873.

Kopriva D (2009) Implementing Spectral Methods for Partial Differential Equations: Algorithms for Scientists and Engineers Scientific Computation. Springer Netherlands.

Lasry JM, Lions PL (2007) Mean field games. Japanese journal of mathematics 2:229-260.

Liberzon D (2011) Calculus of variations and optimal control theory: a concise introduction Princeton University Press.

Longhurst AR, Harrison WG (1989) The biological pump: profiles of plankton production and consumption in the upper ocean. Progress in Oceanography 22:47-123.

Nathan R, Getz WM, Revilla E, Holyoak M, Kadmon R, Saltz D, Smouse PE (2008) A movement ecology paradigm for unifying organismal movement research. Proceedings of the National Academy of Sciences 105:19052-19059. 
Ohman MD, Frost BW, Cohen EB (1983) Reverse diel vertical migration: An escape from invertebrate predators. Science 220:1404-1407.

Okubo A, Levin S (2001) Diffusion and Ecological Problems: Modern Perspectives Springer.

Pinti J, Visser AW (2019) Predator-prey games in multiple habitats reveal mixed strategies in diel vertical migration. The American Naturalist 193:E65-E77.

Sainmont J, Thygesen UH, Visser AW (2013) Diel vertical migration arising in a habitat selection game. Theoretical ecology 6:241-251.

Thygesen UH, Patterson TA (2018) Oceanic diel vertical migrations arising from a predator-prey game. Theoretical Ecology pp. 1-13.

Thygesen UH, Sommer L, Evans K, Patterson TA (2016) Dynamic optimal foraging theory explains vertical migrations of bigeye tuna. Ecology $97: 1852-1861$.

Wirtz K, Smith SL (2020) Vertical migration by bulk phytoplankton sustains biodiversity and nutrient input to the surface ocean. Scientific reports 10:1-12. 